\title{
Optimal State Discrimination Using Particle Statistics
}

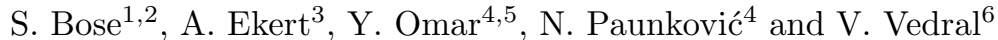 \\ ${ }^{1}$ Institute for Quantum Information, California Institute of Technology, CA 91125 \\ 2 Department of Physics and Astronomy, University College London, Gower Street, London WC1E 6BT, United Kingdom \\ ${ }^{3}$ Centre for Quantum Computation, DAMTP, University of Cambridge, Cambridge CB3 0WA, United Kingdom \\ ${ }^{4}$ Centre for Quantum Computation, Clarendon Laboratory, University of Oxford, \\ Parks Road, Oxford OX1 3PU, United Kingdom \\ ${ }^{5}$ Centro de Física de Plasmas, Instituto Superior Técnico, P-1049-001 Lisbon, Portugal \\ ${ }^{6}$ Optics Section, The Blackett Laboratory, Imperial College, London SW' 2BZ, United Kingdom
}

(September 8, 2003)

We present an application of particle statistics to the problem of optimal ambiguous discrimination of quantum states. The states to be discriminated are encoded in the internal degrees of freedom of identical particles, and we use the bunching and antibunching of the external degrees of freedom to discriminate between various internal states. We show that we can achieve the optimal single-shot discrimination probability using only the effects of particle statistics. We discuss interesting applications of our method to detecting entanglement and purifying mixed states. Our scheme can easily be implemented with the current technology.

Recently, there has been an emerging interest in the use of particle statistics (both bosonic and fermionic) for quantum information processing $[1,2]$. In fact, it was shown that a useful task such as entanglement concentration could be accomplished, even if non-optimally, using only the effects of quantum statistics, without the need for any other interactions [2]. The above investigations differ significantly from some previous suggestions, where either anyonic statistics [3] or the effects of electronic statistics in conjunction with other interactions $[4,5]$ were used for quantum information processing. Schemes using only particle statistics $[1,2]$ would be very useful for tasks implemented with identical particles that interact very weakly or not at all with each other, such as photons or neutrons. This weak interaction can be beneficial for information processing as it may reduce the unwanted coupling to the environment. Such schemes are also extremely general in the sense of being independent of the actual particle species. It is however not known whether such schemes can accomplish quantum information processing efficiently. Here we present a particular quantum information processing task involving two qubits and show that it can be performed optimally using only quantum statistics. Moreover, we point out how the task of discriminating quantum states can be applied to detecting entanglement and purifying mixed states. We also discuss how to generalize these tasks to $N$ qubits and argue that quantum statistics could be used to perform even this generalized task optimally. While the two qubits and other small $N$ qubits versions of our protocol can be tested with photons, electrons, neutrons or atoms, the large number of qubits versions could have interesting implementations in optical lattices $[6,7]$.

One of the striking aspects of quantum mechanics is that it is not possible to perfectly discriminate between two states unless they are orthogonal. Suppose someone prepares two qubits encoded in the internal degrees of freedom of two identical particles - say, in the spin of two electrons or the polarization of two photons - in one of the following two possible states:

- spins aligned (parallel) and pointing in an arbitrary direction;

- spins anti-aligned and pointing in an arbitrary direction.

We will assume that the two states are equally likely for the sake of simplicity, but all the presented results will be valid for any a priori distribution. Note also that, unless otherwise stated, whenever we mention spin we will actually refer to any two-dimensional internal degree of freedom, be it for fermions or for bosons. As we do not have any knowledge of the direction of alignment in both of the above cases, the overall states are mixed. They are described respectively by the following density operators:

$$
\rho_{2}=\frac{1}{4 \pi} \int d \Omega|\Omega\rangle\langle\Omega|\otimes| \Omega\rangle\langle\Omega|,
$$

and

$$
\sigma_{2}=\frac{1}{4 \pi} \int d \Omega|\Omega\rangle\left\langle\Omega|\otimes| \Omega^{\perp}\right\rangle\left\langle\Omega^{\perp}\right| .
$$

Here, the subscripts indicate that we are considering two particles. The ket $|\Omega\rangle$ represent the spin-up state along the axis defined by the angle $\Omega$, while $\left|\Omega^{\perp}\right\rangle$ is the orthogonal state spin-down. Thus, $\rho_{2}$ represents an equal mixture of spins aligned along an arbitrary axis in space, while $\sigma_{2}$ is the equal mixture of anti-aligned spins of two spin- $\frac{1}{2}$ particles. It is impossible to discriminate between these states perfectly because they are not orthogonal.

Optimal results are known for the discrimination of any two given quantum states $\eta$ and $\eta^{\prime}$ [8]. The maximal 
probability of ambiguously discriminating between two a priori equally likely quantum states in a single-shot measurement is given by the Helstrom formula:

$$
P_{H}\left(\eta, \eta^{\prime}\right)=\frac{1}{2}+\frac{1}{4} \operatorname{Tr}\left|\eta-\eta^{\prime}\right| .
$$

We now present a procedure for discriminating between $\rho_{2}$ and $\sigma_{2}$, both for fermions and bosons, based only on the effects of particle statistics. To use these effects we interfere particles at a beam splitter. Here we use beam splitter in a generic sense, referring not only to the common optical element (partially silvered mirror) used with photons, but also to any device presenting an analog behavior for other kinds of particles, as already suggested for electrons [9]. In order to distinguish the states we rely on path measurements that discriminate between bunching and antibunching, and that are performed on our particles after letting them pass simultaneously through a 50/50 beam splitter. Note that in such balanced beam splitters two indistinguishable particles will always bunch if they are bosons [10], and always antibunch if they are fermions [9] (see also [11]).

For fermions, our guess in the case of the antibunching result is that the input state was $\rho_{2}$, while in the case of bunching is that it was $\sigma_{2}$. The probability of success of our procedure is then:

$$
P_{B S}\left(\rho_{2}, \sigma_{2}\right)=\frac{3}{4} .
$$

This probability can easily be calculated by noticing that the only case for which our guess could be incorrect is when we have the antibunching result. Then, we conclude that the input state was $\rho_{2}$, while it could have actually been $\sigma_{2}$. On the other hand, in the case of the bunching result we know for sure that the input state was $\sigma_{2}$, since - according to the Pauli exclusion principle two particles with aligned spins cannot end up in the same output arm of the beam splitter. When our input state is $\sigma_{2}$, the antibunching happens with probability $1 / 2$, giving in total a probability of incorrect inference of $1 / 4$. In the case of bosons, our protocol is exactly the opposite of the fermionic one, but yields precisely the same efficiency: this time the antibunching results stand for $\sigma_{2}$, whereas the bunching ones stand for $\rho_{2}$, but the probability of success coincides with Eq. (4).

Interestingly, the Helstrom formula gives the same result for the maximal probability of discriminating between these two states:

$$
P_{H}\left(\rho_{2}, \sigma_{2}\right)=\frac{3}{4} .
$$

We can thus conclude that our procedure is optimal for both fermions and bosons.

Helstrom's probability can still be achieved in other cases using the effects of quantum statistics. We now introduce a case of special interest that can be applied to other tasks such as entanglement detection and state purification, as will be shown later in the paper. Suppose that we have to discriminate between the following two states:

- spins aligned (parallel) and pointing in an arbitrary direction (the same as in Eq. (1));

- each spin in the maximally mixed state.

The latter state is represented by the following operator:

$\tau_{2}=\frac{1}{4} I^{\otimes 2}=\frac{1}{4}(|0\rangle\langle 0|+| 1\rangle\langle 1|) \otimes(|0\rangle\langle 0|+| 1\rangle\langle 1|)$,

where $|0\rangle$ and $|1\rangle$ are any two orthogonal spin states.

The strategy now is exactly the same as before. It relies on the fact that if the state is $\rho_{2}$ the particles can, due to their indistinguishability, give only one result (antibunching in the case of fermions or bunching in the case of bosons), while if the input state is $\tau_{2}$ both results are possible. This time, the probability of success is:

$$
P_{B S}\left(\rho_{2}, \tau_{2}\right)=\frac{5}{8}
$$

and this coincides with the Helstrom result

$$
P_{H}\left(\rho_{2}, \tau_{2}\right)=\frac{5}{8},
$$

so our procedure is optimal in this case too. The calculations leading to the above results are analogous to the ones in the previous discrimination case.

One of the most interesting applications of our approach can be found in the detection of entanglement. We illustrate this in the case of pure states of two particles. Suppose, for example, that we are to discriminate any maximally entangled state of two qubits from any disentangled (product) state. As before, the qubits are supported by the internal degrees of freedom of two identical particles (labelled, say, $A$ and $B$ ). Suppose in addition that we are given two identical copies of the state. In order to detect entanglement, we take the same particle (either $A$ or $B$ ) from each pair and interfere them at a beam splitter, as shown in Fig. 1. The crux of the argument is that if the state is entangled, then the reduced states of these particles will be maximally mixed as in the state $\tau_{2}$. On the other hand, if the state is separable, then the two interfering particles are in the state $\rho_{2}$. This is the same as in our discrimination procedure above, and so there is a probability of $5 / 8$ to detect entanglement. This example can be generalized to other entangled pure states, and, more interestingly, to more particles. We note that there is a close analogy between this method and our entanglement concentration scheme in [2]. In particular, if the two states were less than maximally entangled, then by detecting entanglement we would actually also amplify it (see [2] for more details). 
Another interesting application is in mixed state purification, as in [12]. Suppose that we start with two qubits, each in some mixed state. We would like to make the state of these qubits purer (in the sense of having lower linear entropy), but also to preserve their original direction in the Bloch sphere. The optimal way of doing so (as proven in [12]) is to project the joint state onto the symmetric subspace, in which case the resulting mixed state is purer and yet preserves the original direction. If the projection is unsuccessful, the qubits are thrown away. This is exactly the same as our probabilistic discrimination with a beam splitter.

We would now like to investigate the generalization of the above results to $N$ particles. For this, we use a generalized (N-port) balanced beam splitter, as shown in Fig. 2, which acts only on the spatial degrees of freedom of the input particles. This action is given by a unitary matrix $U_{N}$, with elements:

$$
u_{m n}=\frac{1}{\sqrt{N}} e^{i \frac{2 \pi}{N}(m-1)(n-1)} .
$$

(Note that there exist alternative descriptions of balanced multiport beam splitters [13].) The square of the norm of each element in the matrix represents the probability that the particle in the $m$-th input arm of the beam splitter ends up in the $n$-th output arm. Since all these elements have norm $\frac{1}{\sqrt{N}}$, we have a representation of a balanced N-port beam splitter.

The aim is now to discriminate between the N-particle generalizations of $\rho_{2}$ and $\tau_{2}$. Those states are given by:

$$
\rho_{N}=\frac{1}{4 \pi} \int d \Omega(|\Omega\rangle\langle\Omega|)^{\otimes N}
$$

and

$$
\tau_{N}=\frac{1}{2^{N}}(|0\rangle\langle 0|+| 1\rangle\langle 1|)^{\otimes N} .
$$

To calculate the Helstrom probability, one has to diagonalize the matrix $\rho_{N}-\tau_{N}$. This turns out to be straightforward once we notice that $\rho_{N}$ can also be represented as an equal mixture of all possible symmetric states within the basis $\left\{\left|S_{i}\right\rangle: i=1, \ldots, N+1=|S|\right\}$ of $N$ qubits. Then, we have:

$$
\rho_{N}=\frac{1}{|S|} \sum_{i=1}^{|S|}\left|S_{i}\right\rangle\left\langle S_{i}\right| .
$$

It is now easy to calculate the Helstrom formula by expanding $\rho_{N}$ in a basis consisting of the union of a basis of the symmetric sub-space and a basis of its orthogonal complement. The result is:

$$
P_{H}\left(\rho_{N}, \tau_{N}\right)=1-\frac{(N+1)}{2^{(N+1)}} .
$$

Alternatively, we can calculate the average probability of success to distinguish states $\rho_{N}$ and $\tau_{N}$ using the following expression:

$$
P_{H}\left(\rho_{N}, \tau_{N}\right)=\frac{1}{2} 1+\frac{1}{2} p .
$$

Here, the $1 / 2$ factors refer to the fact that the two states are prepared with equal probability. The term 1 comes from the fact that the state $\rho_{N}$, supported on the symmetric subspace (of dimension $d_{S}=N+1$ ), is always identified reliably as such, and $p$ is the probability of identifying the other state, $\tau_{N}$. Since $\tau_{N}$ is maximally mixed, it is uniformly distributed over the whole space of $N$ qubits (of dimension $d=2^{N}$ ). In this case $p=\frac{1-d_{S}}{d}$, which after substitution in Eq. (14) gives Eq. (13) right away.

The problem to apply our discrimination scheme to $N$ particles is that it becomes exponentially hard to calculate $P_{B S}\left(\rho_{N}, \tau_{N}\right)$ as $N$ increases. Moreover, it is not clear which inference strategy should be followed. For fermions, the natural generalization seems to be to associate the antibunching results with $\rho_{N}$ and the others with $\tau_{N}$. For bosons, on the other hand, a more subtle strategy may be needed. This is because, loosely speaking, for bosons there is no clear analogue of the Pauli exclusion principle. Furthermore, even without having the complete calculations for $N>2$, we would like to emphasize the remarkable fact that the Helstrom probability $P_{H}\left(\rho_{N}, \tau_{N}\right)$ is equal to the probability of success of a fermionic beam splitter strategy described above, if calculated under the assumption that the particles are classical (i.e., always distinguishable by some arbitrary label), but obey a constraint equivalent to the Pauli exclusion principle (not allowing more than two particles in the same internal state to share the same output arm of the beam splitter). The overall probability is then calculated by summing up the probabilities of all the possible outcomes rather than the amplitudes, as it would be done in the quantum case. However, in the case of three fermions $(N=3)$, we have performed the full quantum calculations (i.e., taking properly into account the effects of statistics) for a three-port balanced beam splitter and obtained:

$$
P_{B S}\left(\rho_{3}, \tau_{3}\right)=\frac{3}{4},
$$

which is equal to $P_{H}\left(\rho_{3}, \tau_{3}\right)$. We believe this result of obtaining the optimal discrimination probability using the effects of particle statistics (in multiports) can be generalized to an arbitrary $N$, both for fermions and bosons, and we continue research in this direction. For now this remains a conjecture. In an optical lattice, with one particle in each lattice site, a multiport beam splitter could probably be simulated by dissolving $\mathrm{N}$ potential wells and then creating a new set of $\mathrm{N}$ wells [7]. Of course, if the particles interact, then the effective beam splitter will be 
modified, and here we only point out the plausibility of creating multiport beamsplitters (or multiparticle interference) in an optical lattice.

In this paper we have shown that it is possible to perform an optimal quantum information processing task using only the effects of particle statistics. In particular, we have presented a strategy for discriminating between two non-orthogonal states of two qubits (encoded in the internal degrees of freedom of identical particles) using beam splitters. We have considered two discrimination scenarios and in each of them our strategy differs (symmetrically) between fermions and bosons, but offers the same efficiency. We also pointed out how our discrimination scheme can be applied to detecting entanglement and purifying mixed states. In addition, we have calculated the Helstrom probability for $N$ qubits in one of our discrimination scenarios. We have shown by explicit calculation that this probability can be achieved in a fermionic three-port beam splitter strategy and that it is the same as the fermionic strategy for general $N$ if the fermions are considered as classical particles that obey the Pauli exclusion principle as the only additional constraint. An advantage of our method is that it can also be easily implemented with the current technology.

Our work suggests a number of interesting research directions. One problem is to prove the optimality of the beam splitter strategy in the case of $N$ qubits and its application to multiparty entanglement detection. This, we hope, will answer the question of weather the symmetry between fermions and bosons in our strategy will be preserved for a generalized beam splitter. It may also lead to a simple and physically intuitive selection principle governing bosonic behavior. Another possible direction is to classify all the pairs of states that can be optimally discriminated with our scheme. Finally, our results suggest that it would be worth to further explore the role of particle statistics in quantum information tasks, in particular in efficient quantum computation.

After the conclusion of this paper, an interesting related work was pointed out to us [14]. The authors would like to thank G. Castagnoli on many useful discussions on the link between computational complexity and particle statistics. His ideas have stimulated much of the work in this paper. We also would like to thank F. A. Bovino and P. Varisco for useful discussions on the role of particle statistics in quantum information processing. S.B. acknowledges support from the NSF under Grant Number EIA-00860368. Y.O. acknowledges support from Fundação para a Ciência e a Tecnologia from Portugal and wishes to thank the Institute for Quantum Information at Caltech for their hospitality. N.P. and V.V. thank Elsag S.p.A. for financial support and Centro de Física de Plasmas at IST in Lisbon for their hospitality. V.V. acknowledges support from Hewlett-Packard company, EPSRC and the European Union projects EQUIP and TOPQIP. This work has been partly supported by the QUIPROCONE collaboration grant number 044.

[1] Y. Omar, N. Paunković, S. Bose and V. Vedral, Phys. Rev. A 65, 062305 (2002).

[2] N. Paunković, Y. Omar, S. Bose and V. Vedral, Phys. Rev. Lett. 88, 187903 (2002).

[3] A. Yu. Kitaev, Annals Phys. 303, 2-30 (2003).

[4] D. Loss and D. P. DiVincenzo, Phys. Rev. A 57, 120 (1998).

[5] A. T. Costa, Jr. and S. Bose, Phys. Rev. Lett. 87, 277901 (2001).

[6] J. Pachos and P. L. Knight, quant-ph/0301084 (2003).

[7] D. Jaksch et al., Phys. Rev. Lett. 82, 1975 (1999).

[8] C. W. Helstrom, Quantum Detection and Estimation Theory, Academic Press, New York (1976).

[9] R. C. Liu, B. Odom, Y. Yamamoto and S. Tarucha, Nature 391, 263 (1998).

[10] C. K. Hong, Z. Y. Ou and L. Mandel, Phys. Rev. Lett. 59, 2044 (1987).

[11] R. Loudon, Phys. Rev. A 58, 4904 (1998).

[12] J. I. Cirac, A. K. Ekert and C. Macchiavello, Phys. Rev. Lett. 82, 4344 (1999).

[13] I. Jex, S. Stenholm and A. Zeilinger, Opt. Commun. 117, 95 (1995); see also M. Żukowski, A. Zeilinger and M. A. Horne, Phys. Rev. A 55, 2564 (1997).

[14] S. M. Barnett, A. Chefles and I. Jex, Phys. Lett. A 307, 189 (2003). 


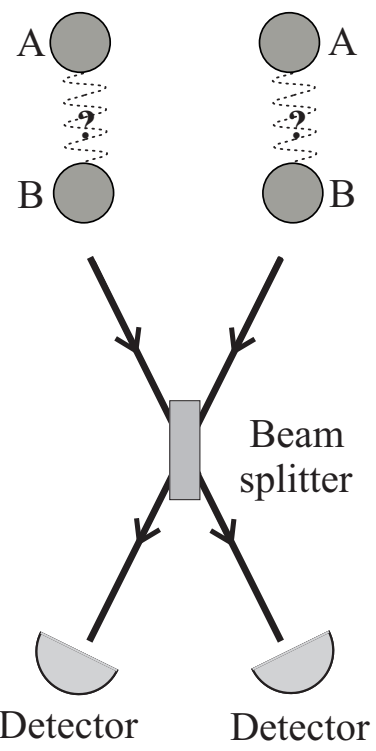

FIG. 1. This figure represents the set-up for our entanglement detection scheme. We have two equal pairs of identical particles and consider their internal degrees of freedom. Both pairs are in the same pure state: either separable or maximally entangled. We take the same particle (for instance, $B$ ) from each pair and interfere them at a 50/50 beam splitter. If the states are disentangled then the particles are indistinguishable and, depending on the statistics, will either only bunch or only antibunch. Otherwise, if the states are maximally entangled, the particles are maximally mixed, meaning that they can be (probabilistically) distinguished and hence the statistics does not influence their behavior. This entanglement detection is a particular instance of our state discrimination scheme discussed in the paper.

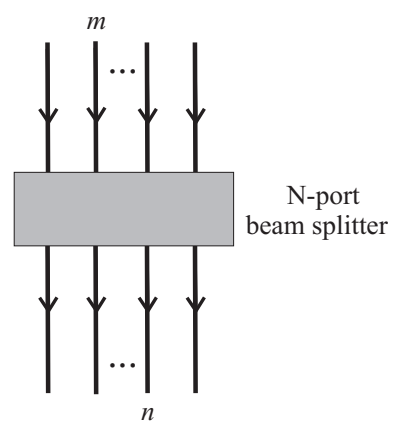

FIG. 2. This diagram represents a multiport beam splitter with $N$ inputs and $N$ outputs. The overall output state depends not only on the input, but also on the statistics (either fermionic or bosonic) of the identical particles involved. We have labelled two arbitrary ports, the $m$-th input port and the $n$-th output port. 